\title{
Study of the heavy molecular states in quark model with meson exchange Interaction
}

\author{
YU Si-Hai ${ }^{1}$ WANG Bao-Kai ${ }^{1}$ CHEN Xiao-Lin ${ }^{2}$ DENG Wei-Zhen ${ }^{1 ; 1)}$ \\ 1 (Department of Physics and State Key Laboratory of Nuclear Physics and Technology, \\ Peking University, Beijing 100871, China) \\ 2 (Department of Physics, Peking University, Beijing 100871, China)
}

\begin{abstract}
Some charmonium-like resonances such as $X(3872)$ can be interpreted as possible $D^{(*)} \bar{D}^{(*)}$ molecular states. Within the quark model, we study the structure of such molecular states and the similar $B^{(*)} \bar{B}^{(*)}$ molecular states by taking into account the light meson exchange $(\pi, \eta, \rho, \omega$ and $\sigma)$ between two light quarks from different mesons.
\end{abstract}

Key words molecular state, quark model, meson exchange

PACS 12.39.Pn, 12.39.Fe, 14.40.Rt

\section{Introduction}

Some recently discovered narrow charmoniumlike resonances have aroused great theoretical interest. The typical $X(3872)$ state, which was discovered by the Belle Collaboration [1] and subsequently confirmed by the CDF Collaboration [2] and BABAR Collaboration [3], is summarized $M_{X}=$ $3872.2 \pm 0.8 \mathrm{MeV}, \Gamma_{X}=3.0_{-1.4}^{+1.9} \pm 0.9 \mathrm{MeV}$ [4]. Previously its quantum numbers were inferred most probably $J^{P C}=1^{++}$, while the masses of the corresponding charmonium states in the quark model $2^{3} P_{1}$ or $3^{3} P_{1}$ are hundreds $\mathrm{MeV}$ above $M_{X}$. Recently, a study of the $\pi^{+} \pi^{-} \pi^{0}$ mass distribution from the $X(3872)$ decay by the BABAR Collaboration favors a negative parity assignment $2^{-+}[5]$. However, still the mass of the charmonium candidate in the quark model $1^{1} D_{2}$ disagrees with $M_{X}$.

Since it is difficult to assign $X(3872)$ to any conventional $c \bar{c}$ charmonium state in the quark model, other alternative explanations prevail. Because $M_{X}$ is close to the $D^{*} \bar{D}$ threshold, $X(3872)$ was interpreted as a loosely bound molecular $D^{*} \bar{D}$ state [6 9] or such a molecular state with some admixtures of $\rho J / \psi$ and $\omega J / \psi[10]$. It was also suggested as a tetra quark state dominated by a diquark-antidiquark structure [11] or a $c \bar{c} g$ hybrid state [12]. The proximity of the $X(3872)$ to $D^{*} \bar{D}$ threshold may also imply that the cusp scenario should be treated seriously [13].
The $D^{(*)} \bar{D}^{(*)}$ molecular states were proposed many years ago 14, 15]. To bind the two mesons together in a molecular state, additional interaction beyond the quark potential model should be introduced between the two mesons. Naturally the meson exchanges were first considered. The inter-meson potentials from light meson exchanges can be easily treated in the frame of the heavy quark effecitive theory (HQET). The studies showed that the cutoff parameters in the form factors are critical to the formation of the molecular states [7, 16 18]. Other interactions such as the gluon exchanges were also considered [10, 19, 20].

Basically, the form factor is related to the inner quark wave functions of the meson state. To avoid the uncertainty from the form factor, in this work we will investigate the possible molecular states directly using the quark wave function within the quark model. Our calculation is based on the multiGaussian function expansion of the quark wave function of the molecular state, which is a rather simple and efficient variational method to study many-body ground bound states 2023 .

We will assume that the light mesons $(\pi, \eta, \rho, \omega$ and $\sigma$ ) are exchanged between the light quarks from different hadrons. The mechanism can be understood from the chiral quark model 24] which was proposed by Weinberg 25] and formulated by Manohar and Georgi [24]. The key feature of the chiral quark model 
is that between the QCD confining scale and the chiral symmetry breaking scale, QCD can be roughly described by an effective Lagrangian of quarks and pseudo-scalar mesons of Numbu-Goldstone bosons. The strong interaction between hadrons is thus described mainly by the exchange of pseudo-scalar mesons at long-range. In practice, the chiral quark model can be further extended with more mesons to account for the intermidiate-range interaction. The light vector resonances $\rho, K^{*}$, etc. can be realized as the dynamical gauge bosons of a hidden local symmetry [26]. The scalar meson $\sigma$ can be explicitly introduced from the linear realization, as in the linear sigma model. Chiral quark model was widely used in the study of nuclear forces [27,28] and recently used to study the molecular states [29].

In section 2, we will introduce the quark model with the meson exchange interactions in our work. In section 3, the multi-Gaussian function expansion method and the configuration space of the molecular states are presented. We will study both the $D^{(*)} \bar{D}^{(*)}$ and $B^{(*)} \bar{B}^{(*)}$ molecular states. Finally we will give a short summary.

\section{The quark model with meson ex- change}

The Hamiltonian in a quark model can be written as

$H=\sum_{i}\left(m_{i}+\frac{P_{i}^{2}}{2 m_{i}}\right)-\frac{3}{4} \sum_{i<j}\left(F_{i} \cdot F_{j} V_{i j}^{c}+F_{i} \cdot F_{j} S_{i} \cdot S_{j} V_{i j}^{s}\right)$.

The first part is the non-relativistic kinetic energy, where $m_{i}$ 's are the constituent quark masses. The second part is the central potential, where $F_{i}=$ $\frac{1}{2} \lambda_{i}$ are the well known $S U(3)_{c}$ Gell-Mann matrices. Apart from a constant, the central potential usually is a combination of the one gluon exchange coulomb potential and the linear confinment:

$$
V_{i j}^{c}=-\frac{\kappa}{r_{i j}}+\frac{r_{i j}}{a_{0}^{2}}-M_{0},
$$

where $r_{i j}=\left|r_{i}-r_{j}\right|$ is the distance between quark $i$ and quark $j$. The last part is the color-magnetic interaction, where $S_{i}=\frac{1}{2} \sigma_{i}$ are the Pauli matrices.
In our work we will use the Bhaduri quark model which is a rather simple non-relativistic quark potential model [30]. The potential function $V_{i j}^{s}$ of colormagnetic interaction reads

$$
V_{i j}^{s}=\frac{4 \kappa}{m_{i} m_{j}} \frac{1}{r_{0}^{2} r_{i j}} e^{-r_{i j} / r_{0}} .
$$

The model parameters are

$$
\begin{array}{ll}
\kappa=102.67 \mathrm{MeV}, & a_{0}=0.0326(\mathrm{fm} / \mathrm{MeV})^{\frac{1}{2}}, \\
M_{0}=913.5 \mathrm{MeV}, & r_{0}=0.4545 \mathrm{fm}, \\
m_{u}=m_{d}=337 \mathrm{MeV}, & m_{s}=600 \mathrm{MeV}, \\
m_{c}=1870 \mathrm{MeV}, & m_{b}=5290 \mathrm{Mev} .
\end{array}
$$

The quark model is very successful in describing the hadron properties at low energy under the QCD confinemnet scale. As is well known, due to the color symmetry, the above quark potential does not provide direct interaction between hadrons. Here we will consider the exchange of the light mesons between the two light quarks from different hadrons based on the chiral quark model. At present, we will consider the exchanges of $\pi, \eta, \omega, \rho$, and $\sigma$ mesons only. The interaction Lagrangian densities are

1. Pseudoscalar

$$
\mathcal{L}_{p}=i g_{p} \bar{\psi}(x) \gamma_{5} \psi(x) \varphi(x),
$$

2. Scalar

$$
\mathcal{L}_{s}=g_{s} \bar{\psi}(x) \psi(x) \phi(x)
$$

3. Vector

$$
\begin{aligned}
\mathcal{L}_{v}= & g_{v} \bar{\psi}(x) \gamma_{\mu} \psi(x) v^{\mu}(x) \\
& +\frac{f_{v}}{2 m_{q}} \bar{\psi}(x) \sigma_{\mu \nu} \psi(x) \partial^{\mu} v^{\nu} .
\end{aligned}
$$

Here $m_{q}$ is the constituent quark mass, $\psi(x)$ is the constituent quark Dirac spinor field. $\varphi(x), \phi(x)$, $v^{\mu}(x)$ are the pseudoscalar, scalar and vector intermediate meson fields respectively. In the case of isovector mesons, the above meson fields should be replaced by $\boldsymbol{\tau} \cdot \boldsymbol{\varphi}, \boldsymbol{\tau} \cdot \boldsymbol{\phi}$ or $\boldsymbol{\tau} \cdot \boldsymbol{v}^{\mu}$ respectively, where $\boldsymbol{\tau}$ are the isospin Pauli matrices. From the effective Lagrangian we can obtain the effective potential between two quarks. In the coordinate space [31],

1. Pseudoscalar

$$
V_{p}(r)=\frac{g_{p}^{2}}{48 \pi} m_{p}\left(\frac{m_{p}}{m_{q}}\right)^{2} Y_{1}\left(m_{p} r\right) \boldsymbol{\sigma}_{1} \cdot \boldsymbol{\sigma}_{2}
$$


2. Scalar

$$
V_{s}(r)=-\frac{g_{s}^{2}}{4 \pi} m_{s}\left[Y\left(m_{s} r\right)-\left(\frac{m_{s}}{m_{q}}\right)^{2} \frac{1}{4} Y_{1}\left(m_{s} r\right)\right]
$$

3. Vector

$$
\begin{aligned}
V_{v}(r)= & \frac{g_{v}^{2}}{4 \pi} m_{v}\left\{Y\left(m_{v} r\right)+\left(\frac{m_{v}}{m_{q}}\right)^{2} \frac{1}{2} Y_{1}\left(m_{v} r\right)\right\}+\frac{g_{v} f_{v}}{4 \pi} m_{v}\left(\frac{m_{v}}{m_{q}}\right)^{2}\left[\frac{1}{2} Y_{1}\left(m_{v} r\right)+\frac{1}{3} Y_{1}\left(m_{v} r\right) \boldsymbol{\sigma}_{1} \cdot \boldsymbol{\sigma}_{2}\right] \\
& +\frac{f_{v}^{2}}{4 \pi} m_{v}\left(\frac{m_{v}}{m_{q}}\right)^{2} \frac{1}{6} Y_{1}\left(m_{v} r\right) \boldsymbol{\sigma}_{1} \cdot \boldsymbol{\sigma}_{2} .
\end{aligned}
$$

We have neglected the momentum dependence of all potentials in the present work. The spin-orbit interaction and the tensor-force are also dropped out here since we only consider the molecular ground states. The functions $Y, Y_{1}$ are defined as follows:

$$
\begin{aligned}
Y(x) & =\frac{e^{-x}}{x} \\
Y_{1}(x) & =\frac{e^{-x}}{x}-4 \pi \delta^{3}(\boldsymbol{x}) .
\end{aligned}
$$

Note that on the quark level we need not introduce the form factors to treat the $\delta$-interaction. The meson's quark wave functions will naturally smear out the singularity.

The $q \bar{q}$ potentials are obtained from the above $q q$ potentials by a $G$-parity transformation. Also there should be an isospin factor $\boldsymbol{\tau}_{1} \cdot \boldsymbol{\tau}_{2}$ in the above effective potential if an isovector meson is exchanged.

From PDG[4], the masses of exchanged mesons are taken to be

$$
\begin{array}{ll}
m_{\pi}=134.98 \mathrm{MeV}, & m_{\eta}=547.85 \mathrm{MeV}, \\
m_{\rho}=775.5 \mathrm{MeV}, & m_{\omega}=782.7 \mathrm{MeV}, \\
m_{\sigma}=600.0 \mathrm{MeV} . &
\end{array}
$$

The masses of relevant heavy flavor mesons are:

$$
\begin{array}{ll}
m_{D^{0}}=1864.5 \mathrm{MeV}, & m_{D^{ \pm}}=1869.3 \mathrm{MeV}, \\
m_{D * 0}=2006.7 \mathrm{MeV}, & m_{D^{* \pm}}=2010.0 \mathrm{MeV}, \\
m_{B^{0}}=5279.5 \mathrm{MeV}, & m_{B^{ \pm}}=5279.1 \mathrm{MeV}, \\
m_{B *}=5325.1 \mathrm{MeV} . &
\end{array}
$$

The coupling constants are taken from Ref. [9]:

$$
\begin{array}{ll}
g_{\pi}=2.74 & g_{\eta}=2.05, \quad g_{\sigma}=3.30, \\
g_{\rho}=3.46, & f_{\rho}=1.45, \\
g_{\omega}=5.28, & f_{\omega}=0,
\end{array}
$$

which are extracted from the meson-nucleon coupling constants in the well-known Bonn model 31] using the single-quark operator approximation [32].

\section{The heavy flavor molecular states}

In this work, we consider the possible molecular states constructed from the pseudo-scalar heavy mesons $(D, B)$ and their vector partners $\left(D^{*}, B^{*}\right)$. The states involving strange mesons are not considered here. The corresponding charmed combinations are: $D-\bar{D}, D^{*}-\bar{D}^{*}, D^{*}-\bar{D}$. Since the charmed mesons belong to the $I=\frac{1}{2}$ representation of isospin $S U(2)$, the possible isospins of the $D^{(*)}-\bar{D}^{(*)}$ system are $I=0$, 1. Following Ref. [33], we label the $D-\bar{D}, D^{*}-\bar{D}$ and $D^{*}-\bar{D}^{*}$ systems as $\Phi_{I J}, \Phi_{I J}^{*}$ and $\Phi_{I J}^{* *}$ respectively, while the $D^{*}-\bar{D}$ system with negative charge conjugate $(C=-1)$ is labeled as $\hat{\Phi}_{I J}^{*}$. Below we pick up the neutral state to represent the isospin multiplet:

1. $I=0$

$$
\begin{aligned}
\Phi_{00}^{0} & =\frac{1}{\sqrt{2}}\left(\bar{D}^{0} D^{0}-D^{-} D^{+}\right) \\
\Phi_{0 J}^{* * 0} & =\frac{1}{\sqrt{2}}\left(\bar{D}^{* 0} D^{* 0}-D^{*-} D^{*+}\right)_{J} \quad(J=0,1,2) \\
\Phi_{01}^{* 0} & =\frac{1}{2}\left[D^{0} \bar{D}^{* 0}-D^{+} D^{*-}\right. \\
& \left.-C\left(\bar{D}^{0} D^{* 0}-D^{-} D^{*+}\right)\right],
\end{aligned}
$$

2. $I=1$

$$
\begin{aligned}
\Phi_{10}^{0} & =\frac{1}{\sqrt{2}}\left(\bar{D}^{0} D^{0}+D^{-} D^{+}\right) \\
\Phi_{1 J}^{* * 0} & =\frac{1}{\sqrt{2}}\left(\bar{D}^{* 0} D^{* 0}+D^{*-} D^{*+}\right) \\
\Phi_{11}^{* 0} & =\frac{1}{2}\left[D^{0} \bar{D}^{* 0}+D^{+} D^{*-}\right. \\
& \left.-C\left(\bar{D}^{0} D^{* 0}+D^{-} D^{*+}\right)\right] .
\end{aligned}
$$$$
\Phi_{1 J}^{* * 0}=\frac{1}{\sqrt{2}}\left(\bar{D}^{* 0} D^{* 0}+D^{*-} D^{*+}\right)_{J} \quad(J=0,1,2)
$$

The states of the $B^{(*)}-\bar{B}^{(*)}$ combinations are constructed similarly but named after $\Omega$.

To calculate the molecular state, we use the Rayleigh-Ritz variation principle. The test wave function is taken to be a series of Gaussian functions with various widths $[21$ 23]. In our case of the $Q \bar{q} q \bar{Q}$ 
molecular state, the test wave function between the $Q \bar{q}$ and $q \bar{Q}$ mesons is expanded to [20]

$$
\begin{aligned}
\psi_{1234}\left(r_{12}, r_{34}, r_{1234}\right)= & \sum_{i} \alpha_{1234}^{i} \psi_{12}\left(r_{12}\right) \psi_{34}\left(r_{34}\right) \\
& \times \exp \left(-\beta_{1234}^{i} r_{1234}^{2}\right),
\end{aligned}
$$

where $\boldsymbol{r}_{1}, \boldsymbol{r}_{2}, \boldsymbol{r}_{3}$ and $\boldsymbol{r}_{4}$ are the coordinates of $Q, \bar{q}, q$ and $\bar{Q}$, respectively. $\boldsymbol{r}_{i j}=\boldsymbol{r}_{i}-\boldsymbol{r}_{j} \cdot r_{1234}$ is the distance between the two meson clusters

$$
\boldsymbol{r}_{1234}=\frac{m_{Q} \boldsymbol{r}_{1}+m_{q} \boldsymbol{r}_{2}}{m_{Q}+m_{q}}-\frac{m_{q} \boldsymbol{r}_{3}+m_{Q} \boldsymbol{r}_{4}}{m_{q}+m_{Q}} .
$$

Each of the meson wave functions $\psi_{i j}\left(r_{i j}\right)$ is also taken to be a Gaussian function series

$$
\psi_{i j}\left(r_{i j}\right)=\sum_{k} \alpha_{i j}^{k} \exp \left(-\beta_{i j}^{k} r_{i j}^{2}\right)
$$

Numerically the above wave function is determined by the variational method in two steps. First the meson wave function (16) is determined from the potential quark model. Then the wave function (14) of the molecular state is obtained from the meson exchange potentials with the meson wave functions $\psi_{i j}$ fixed.

To reduce the amount of computation, the parameters $\beta^{i}$ and $\alpha^{i}$ in each Gaussian function series are determined also in two steps by one-dimensional minimization. We first search a central $\beta$ value using a single Guassian function. Then a set $\left\{\beta^{i}\right\}$ of $2 N+1$ elements is generated by scaling the $\beta$ value up and down by a scale factor $s$ [23]:

$$
\beta^{i}=\beta s^{i-N}
$$

where $i=0,1, \ldots, 2 N$.

The bound energies and the mean squared radii (rms) $\left\langle\boldsymbol{r}_{1234}^{2}\right\rangle^{1 / 2}$ of the $D^{(*)} \bar{D}^{(*)}$ molecular states are listed in Table 1

Table 1. The bound energies of the $D^{(*)} \bar{D}^{(*)}$ molecular states. In calculation II, all the meson coupling constants are reduced by a factor of 0.7 , except the $\pi$ meson.

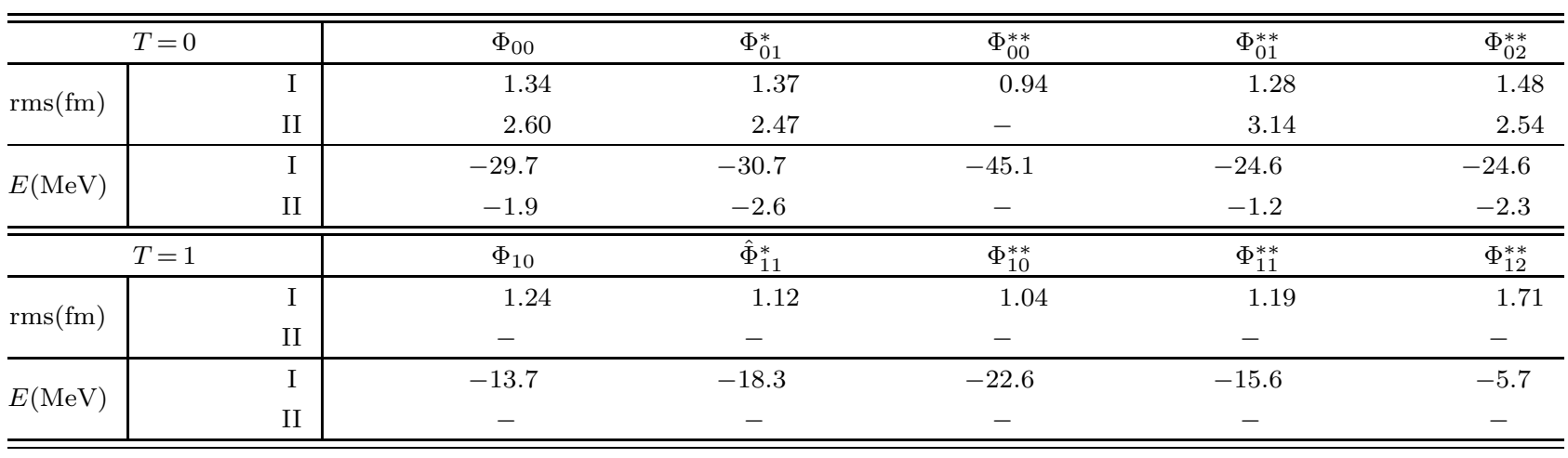

The calculation shows that the meson exchange interaction is strong enough to bind the molecular states. The typical $\Phi_{01}^{*}$ is the candidate molecular state for the $X(3872)$. However the bound energy of $\Phi_{01}^{*}$ is $30.7 \mathrm{MeV}$, which is too larger than what we expect, for $X(3872)$ should be a loose bound molecular state.

However, there is some uncertainty in the meson coupling constants on the quark level. In the Bonn model for neucleon interaction, the form factors 31]

$$
F_{\alpha}\left(\boldsymbol{k}^{2}\right)=\left(\frac{\Lambda_{\alpha}^{2}-m_{\alpha}^{2}}{\Lambda_{\alpha}^{2}+\boldsymbol{k}^{2}}\right)^{n_{\alpha}}
$$

are also introduced in the description of the meson baryon couplings. Clearly from the meson mass de- pendence in the form factor, the effective meson coupling constants decrease as the mass of intermediate mesons increase.

Next we try to decrease the coupling constants to the mesons $\sigma, \rho, \omega$ and $\eta$ with heavier masses by a factor $\lambda$ following Ref [9]. The numerical results for the case of $\lambda=0.7$ are shown in Table 1 as calculation II. Now the bound energy of $\Phi_{01}^{*}$ is only $2.6 \mathrm{MeV}$ and the rms is $2.47 \mathrm{fm}$, which meet the interpretation of $X(3872)$ as a loose bound molecular state. Other possible molecular states left are iso-scalar $\Phi_{00}\left(0^{++}\right)$, $\Phi_{01}^{* *}\left(1^{++}\right)$and $\Phi_{02}^{* *}\left(2^{++}\right)$.

The similar calculation to $B^{(*)} \bar{B}^{(*)}$ molecular states are shown in Table 2 .

Table 2. The bound energies of $B^{(*)} \bar{B}^{(*)}$ molecular states. In calculation II, all the meson coupling constants except the $\pi$ meson are scaled by a factor of 0.7 . 


\begin{tabular}{|c|c|c|c|c|c|c|}
\hline \multicolumn{2}{|c|}{$T=0$} & $\Omega_{00}$ & $\Omega_{01}^{*}$ & $\Omega_{00}^{* *}$ & $\Omega_{01}^{* *}$ & $\Omega_{02}^{* *}$ \\
\hline \multirow{2}{*}{$\operatorname{rms}(\mathrm{fm})$} & I & 1.13 & 1.16 & 0.65 & 1.00 & 1.21 \\
\hline & II & 1.39 & 1.32 & 1.11 & 1.38 & 1.42 \\
\hline \multirow{2}{*}{$E(\mathrm{MeV})$} & I & -46.0 & -49.2 & -81.0 & -44.6 & -44.0 \\
\hline & II & -13.1 & -17.3 & -11.7 & -9.5 & -15.3 \\
\hline \multicolumn{2}{|c|}{$T=1$} & $\Omega_{10}$ & $\hat{\Omega}_{11}^{*}$ & $\Omega_{10}^{* *}$ & $\Omega_{11}^{* *}$ & $\Omega_{12}^{* *}$ \\
\hline \multirow{2}{*}{$\operatorname{rms}(\mathrm{fm})$} & I & 0.72 & 0.66 & 0.62 & 0.68 & 0.84 \\
\hline & II & 1.01 & 0.90 & 0.84 & 0.93 & 1.29 \\
\hline \multirow{2}{*}{$E(\mathrm{MeV})$} & I & -38.6 & -48.9 & -59.3 & -46.6 & -25.6 \\
\hline & II & -10.4 & -14.8 & -19.3 & -13.7 & -5.2 \\
\hline
\end{tabular}

As we expect, the bound energies of $B^{(*)} \bar{B}^{(*)}$ molecular states become larger. Even when the coupling constants are weakened by the scale factor $\lambda=0.7$ in calculation II, the meson exchange is still strong enough to bind the molecular states for all $B^{(*)}-\bar{B}^{(*)}$ combinations.

\section{Summary}

Based on the meson exchange interaction between light quarks, we have investigated the heavy molecular states in quark model. The molecular states are described by the four quarks wave function expanded as a series of Gaussian functions. The numerical results show that the light meson exchanges of $\pi, \eta, \rho, \omega$ and $\sigma$ between the light $u, d$ quarks are strong enough to bind the heavy molecular states.

However, the bound energies of the molecular states are tens of $\mathrm{MeV}$ (up to $80 \mathrm{MeV}$ in $B^{(*)} \bar{B}^{(*)}$ cases) if we adopt the meson-quark coupling constants from the meson-nucleon coupling constants simply using the single-quark operator approxima- tion. The results are unreliable as the bound energies are somehow close to $\Lambda_{\mathrm{QCD}}$ while only the long-range meson exchanges are considered in our calculation.

After we consider the uncertainty of the coupling constants which are deduced from the Bonn potential of neucleon interaction by decreasing the $\eta, \rho, \omega$ and $\sigma$ couplings by a factor of 0.7 , the $X(3872)$ is well interpreted as a loose molecular $1^{++}$state. The calculation also shows that other possible molecular states such as $1^{++}$and $2^{++}$may exist on the threshold of $D^{*} \bar{D}^{*}$ (The $0^{++}$scalar state on the threshold of $D \bar{D}$ is complicated from the scalar admixture). Since the bind energies of $B^{(*)} \bar{B}^{(*)} \mathrm{W}$ molecular states increase with the the mass increase of heavy favor, there are more such molecular states near the $B^{(*)} \bar{B}^{(*)}$ thresholds as we expect.

The main uncertainty in the work is on the estimation of meson-quark coupling constants. To obtain a set of reliable coupling constants, we can use the model to study the nucleon-nucleon interaction in the future.

We would like to thank professor Shi-Lin Zhu for useful discussions.

\section{References}

1 Choi S K et al (Belle). Phys. Rev. Lett., 2003, 91: 262001, http://arxiv.org/abs/hep-ex/0309032 arXiv:hep-ex/0309032

2 Acosta D E et al (CDF II). Phys. Rev. Lett., 2004, 93: 072001, http://arxiv.org/abs/hep-ex/0312021 arXiv: hep-ex/

3 Aubert B et al (BABAR). Phys. Rev., 2005, D71: 071103, http://arxiv.org/abs/hep-ex/0406022 arXiv: hep-ex/0406022 10

4 Amsler C et al (Particle Data Group). Phys. Lett., 2008, B667: 1

5 del Amo Sanchez P et al (BABAR). Phys. Rev., 2010, D82: 011101, http://arxiv.org/abs/1005.5190 arXiv: 1005.5190 [hep-ex]

6 Tornqvist N A. Phys. Lett., 2004, B590: 209-215, http://arxiv.org/abs/hep-ph/0402237arXiv:hep-ph/0402237

7 Liu X, Luo Z G, Liu $\quad$ Y $\quad$ R, Zhu S L. Eur. Phys. J., 2009, C61: 411-428, http://arxiv.org/abs/0808.0073 arXiv:0808.0073 [hep-ph]

8 Thomas C E, Close F E. Phys. Rev., 2008, D78: 034007, http://arxiv.org/abs/0805.3653 arXiv: 0805.3653 [hep-ph]

9 Ding G J, Liu J F, Yan M L. Phys. Rev., 2009, D79:

312934005 , http://arxiv.org/abs/0901.0426 arXiv : 0901.0426 [hep-ph]

10 Swanson E S. Phys. Lett., 2004, B588: 189-195, http://arxiv.org/abs/hep-ph/0311229 arXiv:hep-ph/0311229

11 Maiani L, Piccinini F, Polosa A D, Riquer V. Phys. Rev., 2005, D71: 014028, http://arxiv.org/abs/hep-ph/0412098 arXiv:hep-ph/0412098

12 Li B A. Phys. Lett., 2005, B605: 306-310, http://arxiv.org/abs/hep-ph/0410264 arXiv: hep-ph/0410264

13 Bugg D V. Phys. Lett., 2004, B598: 8-14, http://arxiv.org/abs/hep-ph/0406293 arXiv:hep-ph/0406293

14 De Rújula A, Georgi H, Glashow S L. Phys. Rev. Lett., 
1977, 38: 317

15 Tornqvist N A. Phys. Rev. Lett., 1991, 67: 556-559

16 Suzuki M. Phys. Rev., 2005, D72: 114013

17 Meng C, Gao Y J, Chao K T.http://arxiv.org/abs/ar

18 Zhu S L. Int. J. Mod. Phys., 2008, E17: 283-322

19 Wong C Y. Phys. Rev., 2004, C69: 055202, http://arxiv.org/abs/hep-ph/0311088arXiv:hep-ph/0311088 30

20 Wang B K, Deng W Z, Chen X L. Chinese Physics, 2010, C34: 1052, http://arxiv.org/abs/0910.4787 arXiv: 0910.47873 [hep-ph]

21 Kameyama H, Kamimura M, Fukushima Y. Phys. Rev., 1989, C40: 974-987

22 Varga K, Suzuki Y. Phys. Rev., 1996, A53: 1907-1910

23 Brink D M, Stancu F. Phys. Rev., 1998, D57: 6778-6787

24 Manohar A, Georgi H. Nucl. Phys., 1984, B234: 189

25 Weinberg S. Physica, 1979, A96: 327
26 Georgi H. Nucl. Phys., 1990, B331: 311-330

27 Zhang Z Y et al. Nucl. Phys., 1997, A625: 59-70

28 Dai L R, Zhang Z Y, Yu Y W, Wang

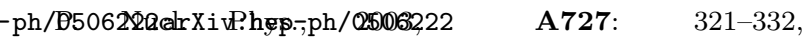
http://arxiv.org/abs/nucl-th/0404004arXiv:nucl-th/0404004

29 Liu Y R, Zhang Z Y. Phys. Rev., 2009, C79: 035206

Bhaduri R K, Cohler L E, Nogami Y. Nuovo Cim., 1981, A65: $376-390$

1 Machleidt R, Holinde K, Elster C. Phys. Rept., 1987, 149: $1-89$

32 Riska D O, Brown G E. Nucl. Phys., 2001, A679: 577-596, http://arxiv.org/abs/nucl-th/0005049arXiv:nucl-th/0005049

33 Liu Y R, Liu X, Deng W Z, Zhu S L. Eur. Phys. J., 2008, C56: $63-73$, http://arxiv.org/abs/0801.3540 arXiv: 0801.3540 [hep-ph] 